\title{
Attributable deaths of liver cancer in China
}

\author{
Maomao Cao ${ }^{*}$, Chao Ding ${ }^{2 *}$, Changfa Xia ${ }^{1}, \mathrm{He} \mathrm{Li}^{1}$, Dianqin Sun ${ }^{1}$, Siyi He${ }^{1}$, Wanqing Chen ${ }^{1}$ \\ ${ }^{1}$ Office of Cancer Screening, National Cancer Center/National Clinical Research Center for Cancer/Cancer Hospital, Chinese Academy of Medical \\ Sciences and Peking Union Medical College/Chinese Academy of Medical Sciences Key Laboratory for National Cancer Big Data Analysis and \\ Implement, Beijing 100021, China; ${ }^{2}$ Department of Anesthesiology, National Cancer Center/National Clinical Research Center for Cancer/Cancer \\ Hospital, Chinese Academy of Medical Sciences and Peking Union Medical College, Beijing 100021, China \\ *These authors contributed equally to this work. \\ Correspondence to: Prof. Wanqing Chen. Office of Cancer Screening, National Cancer Center/National Clinical Research Center for Cancer/Cancer \\ Hospital, Chinese Academy of Medical Sciences and Peking Union Medical College/Chinese Academy of Medical Sciences Key Laboratory for \\ National Cancer Big Data Analysis and Implement, Beijing 100021, China. Email: chenwq@cicams.ac.cn.
}

\begin{abstract}
Objective: There is little information about contributions of the well-known risk factors to the liver cancer burden. We conducted a comparative study to estimate the liver cancer burden attributable to major risk factors.

Methods: Liver cancer deaths for adults were estimated from 978 county-level surveillance points in China in 2014. Risk factors were identified from the International Agency for Research on Cancer and the World Cancer Research Fund International. Population attributable fraction (PAF) by age, sex, and province was calculated using multiple formulas.

Results: In total, $72.4 \%$ of liver cancer deaths could be attributable to the studied risk factors. Hepatitis B virus $(\mathrm{HBV})$ was responsible for the largest fraction of liver cancer burden in both genders $(\mathrm{PAF}=55.6 \%$ in males, $\mathrm{PAF}=46.5 \%$ in females). PAFs for liver cancer burden attributable to smoking (15.7\% vs. 4.8\%), and alcohol drinking $(10.3 \%$ vs. $1.6 \%)$ were significantly higher in males than in females. The burden of HBV-attributable deaths was the highest in Qinghai province.

Conclusions: HBV still contributes to the majority of liver cancer burden than any other risk factors. Targeted preventive measures should be implemented based on the degree of contributions of risk factors to liver cancer deaths.
\end{abstract}

Keywords: Liver cancer; population attributable fraction; comparative study; China

Submitted Jul 21, 2021. Accepted for publication Aug 13, 2021.

doi: 10.21147/j.issn.1000-9604.2021.04.05

View this article at: https://doi.org/10.21147/j.issn.1000-9604.2021.04.05

\section{Introduction}

Liver cancer is the sixth most commonly diagnosed cancer and the third cancer-related death worldwide, with 905,677 cases and 830,180 deaths in 2020. Of these, nearly $45.3 \%$ of new cases and $47.1 \%$ of deaths occurred in China (1). Several epidemiological studies have identified several risk factors associated with liver cancer, including chronic infection with hepatitis $\mathrm{B}$ virus (HBV) or hepatitis $\mathrm{C}$ virus (HCV), alcohol consumption, tobacco use, metabolic diseases such as obesity and diabetes (2-4). Of which, HBV and $\mathrm{HCV}$, primary risk factors for liver cancer in China, have contributed to the most substantial proportion of liver cancer burden in China. Studies have also shown that liver cancer burden varies significantly across regions and sex due to the heterogeneous exposure of risk factors (5).

The population attributable fraction (PAF) is an important epidemiologic metric, which could quantify the proportion that how many cancers could be avoided by eliminating a given risk factor (6). Thus, PAF was defined as the amount of the cancer burden in the target population attributable to a risk factor. In 2013, Fan et al. have 
estimated the PAF for liver cancer attributable to HBV, $\mathrm{HCV}$, aflatoxin exposure, alcohol drinking, and smoking in China using outdated data (7). However, the evidence was limited because they ignored the exposure differences of risk factors on the age group, gender, and geographical level, and the contribution of risk factors to the liver cancer burden may have changed with time. The results need to further update and refine.

We previously conducted a comparative study to estimate the proportion of cancer burden attributable to 23 risk factors by cancer sites and provinces in China. In this study, we aimed to provide detailed results of the liver cancer burden in the Chinese population attributable to $\mathrm{HBV}, \mathrm{HCV}$, diabetes, excess bodyweight, alcohol drinking, tobacco smoking, and Clonorchis sinensis in 2014 stratified by age groups, sex, and provinces.

\section{Materials and methods}

\section{Liver cancer death estimation}

The detailed methods to estimate liver cancer deaths could be found in the study conducted by Chen et al. (8). Briefly, the liver cancer mortality rate in our study was estimated by sex, age group, and province based on 978 cancer registries of 2,859 counties in China in 2014. The age groups were divided into 20-24 years, 25-29 years, 30-34 years, 35-39 years, $40-44$ years, $45-49$ years, $50-54$ years, $55-59$ years, 60-64 years, 65-59 years, and 70 years and older. Liver cancer cases were identified using the International Classification of Diseases for Oncology, third edition.

\section{Potentially modifiable risk factors}

Liver cancer was the accumulative result of exposure to the associated risk factors over many years. We named this period as lag time, and the average time of $10-15$ years for most risk factors was acceptable (9). Therefore, data on the prevalence of selected risk factors were given in 2000-2005. If the information on the prevalence of risk factors was not available during this period, we would allow extending five years of the predefined time boundary. We included the potentially modifiable risk factors of liver cancer in our study when the association of the targeted risk factors and liver cancer was identified, and the exposure data for the risk factors were available. The potentially modifiable risk factors were primarily identified from the monographs of the International Agency for Research on Cancer, metaanalyses and the report from the World Cancer Research
Fund International (10). Given the major risk factors of liver cancer in China, the following risk factors were included in the analysis: HBV, HCV, smoking, alcohol drinking, excess bodyweight, diabetes, and Clonorchis sinensis.

\section{Exposure data collection}

The prevalence of HBV and HCV was extracted from the infectious diseases routine reporting system (http://www. phsciencedata.cn/Share/). According to the seroepidemiological study conducted in 2006, the overall prevalence of hepatitis B surface antigen by sex across China was estimated to be $8.6 \%$ in males and $5.7 \%$ in females (11). The prevalence of anti-HCV in China among males and females was $0.46 \%$ and $0.40 \%$, respectively (12). The estimated prevalence of $\mathrm{HBV}$ and $\mathrm{HCV}$ was thus raked (ie. scaled along multiple dimensions) so that the sum of all provinces was equivalent to the national representative prevalence.

The prevalence data of age-specific, sex-specific, and province-specific of alcohol drinking, smoking, excess bodyweight, diabetes were obtained from the 2002 Chinese National Nutrition Health Survey. Briefly, the survey covered more than 270 thousand persons in 31 provinces, autonomous regions and municipalities under the central government supervision, excepting Hong Kong, Macao, and Taiwan, China, using the stratified multi-stage sampling design $(13,14)$. Alcohol consumption was defined as drinking alcohol on average once a week. Types of alcohol (beer, wine, distilled spirit) or drinking patterns (regular $v$ s. binge drinking) were not considered. Smokers were defined as persons who had ever smoked for at least 6 months. Current smokers were smoking cigarettes within $30 \mathrm{~d}$ before the date of the survey. Information on alcohol and smoking was obtained by well-trained staff using a structured family interview with the predefined health behavior questionnaire. Body mass index was calculated as weight in kilograms divided by height in meter squared. Diabetes was diagnosed for a fasting plasma glucose concentration to $7.0 \mathrm{mmol} / \mathrm{L}$ and above, or an oral glucose tolerance test blood glucose of $11.1 \mathrm{mmol} / \mathrm{L}$ or higher, identified by the World Health Organization diagnostic criteria (1999) (15). The prevalence of Clonorchis sinensis was extracted from a recent publication (16).

\section{Relative risks (RRs)}

RRs for liver cancer and each risk factor were retrieved 
from recent high-quality meta-analyses in China that adopted original articles of case-control and cohort studies with large-scale surveys of representative samples of China. If such studies were not available, non-representative samples of Chinese population studies were used. If Chinese data were not available, the final option was to use meta-analyses from other Asian or non-Asian countries. We abstracted RRs separately for males and females.

\section{Statistical analysis}

A comparative risk assessment was conducted to estimate the liver cancer burden attributable to interested risk factors. The detailed methodology can be found elsewhere (8). Generally, the estimation of PAF relied on the RR of a given risk factor and the prevalence of exposure to a particular risk factor in the total population $(P)$. For the category variable, the general formula is:

$$
P A F=\frac{\sum_{i=1}^{n} P_{i}\left(R R_{i}-1\right)}{\sum_{i=1}^{n} P_{i}\left(R R_{i}-1\right)+1}
$$

where $R R_{i}$ is the RR for exposure category $i, P_{i}$ is the fraction of the population in exposure category $i$, and $n$ is the number of exposure categories (17). For continuous risk factors (excess bodyweight), the following formula, suggested by the Comparative Risk Assessment Collaborative Group, was used to calculate PAF.

$$
P A F=\frac{\int_{x=0}^{m} R R(x) P 1(x) d x-\int_{x=0}^{m} R R(x) P 2(x) d x}{\int_{x=0}^{m} R R(x) P 1(x) d x}
$$

where $R R(x)$ is the RR at exposure level $\mathrm{x}, P l(x)$ is the population distribution of excess bodyweight, $P 2(x)$ is the counterfactual distribution of theoretical minimum risk exposure, and $\mathrm{m}$ the maximum exposure level (17). The two-sided $95 \%$ confidence interval (95\% CI) for the PAF values was estimated using a bootstrap simulation method with 5000 simulations.

\section{Results}

\section{Estimation of exposure and selection of risk estimates}

Overall, in 2014, 233,451 and 85,247 deaths in males and females due to liver cancer in China were estimated. Nationally, there were 230,856 liver cancer deaths attributable to the seven risk factors in both sexes, with a PAF of $72.4 \%$. The estimated sex-specific PAFs of each risk factor for liver cancer are shown in Table 1 . HBV ranked the highest attributable fraction among all risk factors in both sexes and combined. In males, HBV was responsible for 129,894 deaths in 2014 with a PAF of $55.6 \%$, while in females, the corresponding deaths were 39,660 with a PAF of $46.5 \%$. And $17.4 \%$ and $15.9 \%$ of liver cancer deaths can be attributed to HCV in males and females, respectively. PAFs of smoking and alcohol drinking were significantly higher in males $(15.7 \%$ vs. $10.3 \%$ correspondingly) than that in females $(4.8 \%$ vs. $1.6 \%$, correspondingly). However, females had a higher excess bodyweight attributable fraction ( $\mathrm{PAF}=13.0 \%)$ compared to males $(\mathrm{PAF}=10.3 \%)$. Around 33,011 deaths of liver cancer can be attributed to diabetes, and the fraction of liver cancer deaths attributable to diabetes was higher in males than that in females.

\section{Provincial PAF estimation}

Overall PAF for all risk factors and HBV estimations across provinces are shown in Figure 1. Liver cancer deaths can be

\begin{tabular}{|c|c|c|c|c|c|c|c|c|c|}
\hline \multirow{2}{*}{ Risk factors } & \multicolumn{3}{|c|}{ All } & \multicolumn{3}{|c|}{ Males } & \multicolumn{3}{|c|}{ Females } \\
\hline & PAF (\%) & $95 \% \mathrm{Cl}$ & No. of deaths & PAF (\%) & $95 \% \mathrm{Cl}$ & No. of deaths & PAF (\%) & $95 \% \mathrm{Cl}$ & No. of deaths \\
\hline HBV & 53.2 & $50.5-56.2$ & 169,554 & 55.6 & $53.1-58.4$ & 129,894 & 46.5 & $43.4-50.1$ & 39,660 \\
\hline $\mathrm{HCV}$ & 17.0 & $12.7-21.5$ & 54,167 & 17.4 & $13.1-21.8$ & 40,575 & 15.9 & $11.6-20.5$ & 13,592 \\
\hline Smoking & 12.8 & $12.5-13.1$ & 40,751 & 15.7 & $15.4-16.0$ & 36,692 & 4.8 & $4.3-5.2$ & 4,059 \\
\hline Diabetes & 10.4 & $9.3-11.4$ & 33,011 & 10.9 & $9.8-12.1$ & 25,515 & 8.8 & $7.9-9.6$ & 7,496 \\
\hline Excess bodyweight & 11.0 & $10.6-11.4$ & 35,078 & 10.3 & $9.9-10.7$ & 24,014 & 13.0 & $12.5-13.5$ & 11,064 \\
\hline Alcohol drinking & 7.9 & $7.7-8.2$ & 25,289 & 10.3 & $10.0-10.5$ & 23,946 & 1.6 & $1.4-1.7$ & 1,343 \\
\hline Clonorchis sinensis & 0.2 & $0.2-0.3$ & 750 & 0.2 & $0.2-0.2$ & 535 & 0.3 & $0.2-0.3$ & 215 \\
\hline All & 72.4 & $68.8-76.0$ & 230,856 & 75.7 & $72.5-78.9$ & 176,742 & 63.5 & $58.8-68.2$ & 54,114 \\
\hline
\end{tabular}

Table 1 Fraction and number of attributable liver cancer deaths according to risk factors by sex

$\mathrm{HBV}$, hepatitis $\mathrm{B}$ virus; $\mathrm{HCV}$, hepatitis $\mathrm{C}$ virus; $\mathrm{PAF}$, population attributable fraction; $95 \% \mathrm{Cl}, 95 \%$ confidence interval. 
attributable to the included risk factors the most in Qinghai province, followed by Xinjiang and Gansu province. Liver cancer deaths associated with the studied risk factors were mostly distributed in Northwestern China. Similar to the distribution of PAF of overall risk factors, Qinghai province, Xinjiang, and Gansu provinces also had the largest HBV attributable fraction.

\section{Provincial PAF estimation by sex}

PAF estimations stratified by province in males and females are shown in Table 2,3. In males, liver cancer burden attributable to HBV has the highest proportion in Qinghai province, followed by Xinjiang and Gansu province. HCV and excess bodyweight were responsible for the largest liver cancer burden in Xinjiang and Tianjin, respectively. The largest liver cancer burden attributable to diabetes could be found in Tianjin and Shanghai. Smoking was an important contributor to the liver cancer burden in Guizhou province. Alcohol drinking was responsible for 14.4\%, $13.6 \%$, and $13.5 \%$ of liver cancer burden in Tibet, Jiangxi, and Guangxi province, respectively, whereas in females, liver cancer burden mainly attributable to alcohol drinking was observed in Tibet (9.0\%), Jiangxi province (6.6\%) and Guizhou province (4.9\%). The heaviest liver cancer burden attributable to Clonorchis sinensis was in Heilongjiang, Guangdong, and Guangxi provinces in both sexes.

\section{$P A F$ estimation by age group}

Figure 2 depicts PAFs of each risk factor by age group. In males, the fraction of liver cancer deaths attributable to

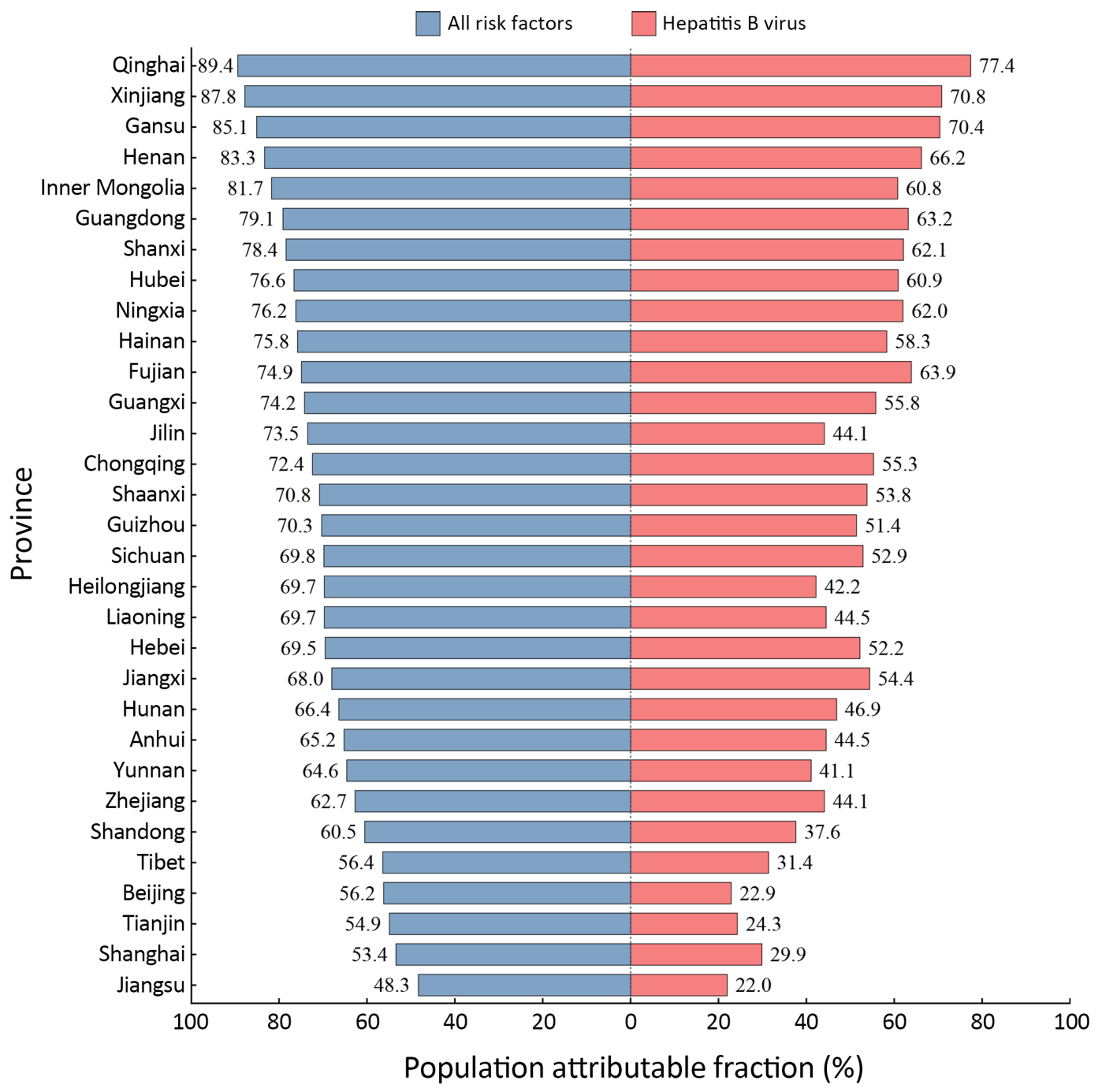

Figure 1 Proportion of liver cancer deaths attributable to all risk factors and Hepatitis B virus, separately. 
Table 2 Provincial-level PAF for liver cancer deaths by selected risk factors in males (\%)

\begin{tabular}{|c|c|c|c|c|c|c|c|c|c|}
\hline Provinces & $\mathrm{HBV}$ & $\mathrm{HCV}$ & Excess bodyweight & Diabetes & Smoking & Alcohol drinking & Clonorchis sinensis & All & $95 \% \mathrm{Cl}$ \\
\hline Beijing & 24.6 & 11.9 & 17.4 & 15.2 & 14.6 & 12.9 & 0 & 59.4 & $51.9-66.6$ \\
\hline Tianjin & 26.0 & 6.6 & 17.8 & 18.9 & 15.0 & 9.5 & 0 & 57.4 & $47.9-66.7$ \\
\hline Shanxi & 64.9 & 24.5 & 10.3 & 10.2 & 17.2 & 7.9 & 0 & 82.1 & $78.7-85.7$ \\
\hline Inner Mongolia & 62.8 & 27.0 & 8.2 & 12.2 & 18.0 & 13.5 & 0 & 83.5 & $78.1-88.4$ \\
\hline Jilin & 46.6 & 29.7 & 11.4 & 13.4 & 15.1 & 11.7 & 0.4 & 76.0 & $69.6-82.3$ \\
\hline Heilongjiang & 44.4 & 19.1 & 13.3 & 11.5 & 15.5 & 13.0 & 1.0 & 71.7 & $65.6-78.3$ \\
\hline Shanghai & 32.3 & 6.6 & 12.6 & 16.9 & 13.1 & 8.6 & 0 & 58.6 & $50.5-65.9$ \\
\hline Jiangsu & 23.8 & 4.8 & 10.3 & 9.4 & 17.0 & 11.8 & 0 & 52.8 & $45.8-59.7$ \\
\hline Zhejiang & 46.3 & 6.9 & 9.1 & 10.3 & 14.8 & 10.6 & 0 & 66.8 & $60.1-73.1$ \\
\hline Shandong & 39.9 & 4.4 & 15.2 & 15.2 & 14.9 & 12.4 & 0 & 64.7 & $58.3-70.5$ \\
\hline Henan & 69.0 & 35.0 & 10.0 & 11.1 & 15.7 & 7.2 & 0 & 86.3 & $83.5-88.9$ \\
\hline Hubei & 63.5 & 14.4 & 9.3 & 10.5 & 15.2 & 12.2 & 0.5 & 79.7 & $75.3-83.9$ \\
\hline Hunan & 49.2 & 16.0 & 5.3 & 5.6 & 16.2 & 10.7 & 0.1 & 70.1 & $64.4-75.9$ \\
\hline Guangdong & 65.0 & 23.0 & 5.3 & 8.4 & 16.8 & 7.6 & 0.8 & 81.4 & $77.7-84.7$ \\
\hline Guangxi & 57.7 & 24.3 & 3.2 & 2.1 & 13.3 & 13.5 & 0.6 & 76.9 & $72.4-81.1$ \\
\hline Hainan & 60.4 & 22.0 & 11.9 & 9.3 & 13.2 & 6.6 & 0.1 & 78.4 & $72.8-83.9$ \\
\hline Chongqing & 57.5 & 12.0 & 9.8 & 10.8 & 16.7 & 11.2 & 0 & 75.8 & $71.0-80.6$ \\
\hline Qinghai & 79.2 & 35.2 & 10.3 & 14.9 & 15.8 & 8.7 & 0 & 91.3 & $88.7-93.8$ \\
\hline Ningxia & 64.6 & 13.3 & 15.3 & 11.0 & 13.1 & 6.6 & 0 & 78.8 & $74.0-83.3$ \\
\hline Xinjiang & 72.9 & 44.5 & 12.4 & 5.8 & 11.9 & 7.6 & 0 & 89.2 & $86.7-91.6$ \\
\hline All & 55.6 & 17.4 & 10.3 & 10.9 & 15.7 & 10.3 & 0.2 & 75.7 & $72.5-78.9$ \\
\hline
\end{tabular}

PAF, population attributable fraction; HBV, hepatitis B virus; $\mathrm{HCV}$, hepatitis $\mathrm{C}$ virus; $95 \% \mathrm{Cl}$, $95 \%$ confidence interval.

$\mathrm{HBV}$ was the largest across all age groups. However, a downtown trend was observed for individuals who were 65 years of age or older. PAF of smoking and alcohol drinking for liver cancer presented decreased trends with age (Figure $2 A$ ). In females, we found that the largest attributable burden was also associated with $\mathrm{HBV}$ among all age groups. Consistent with the trend of PAF of HCV for liver cancer burden in males, the pattern of PAF of $\mathrm{HCV}$ in females also grew with age. PAFs of diabetes, alcohol drinking, and smoking were higher in the old age population (over 60 years) than individuals at a young age (less than 60 years) (Figure $2 B$ ).

\section{Discussion}

Quantifying the disease burden attributable to major risk factors is very important to disease prevention and control. In this study, we estimated the liver cancer burden 
Table 3 Provincial-level PAF for liver cancer deaths by selected risk factors in females (\%)

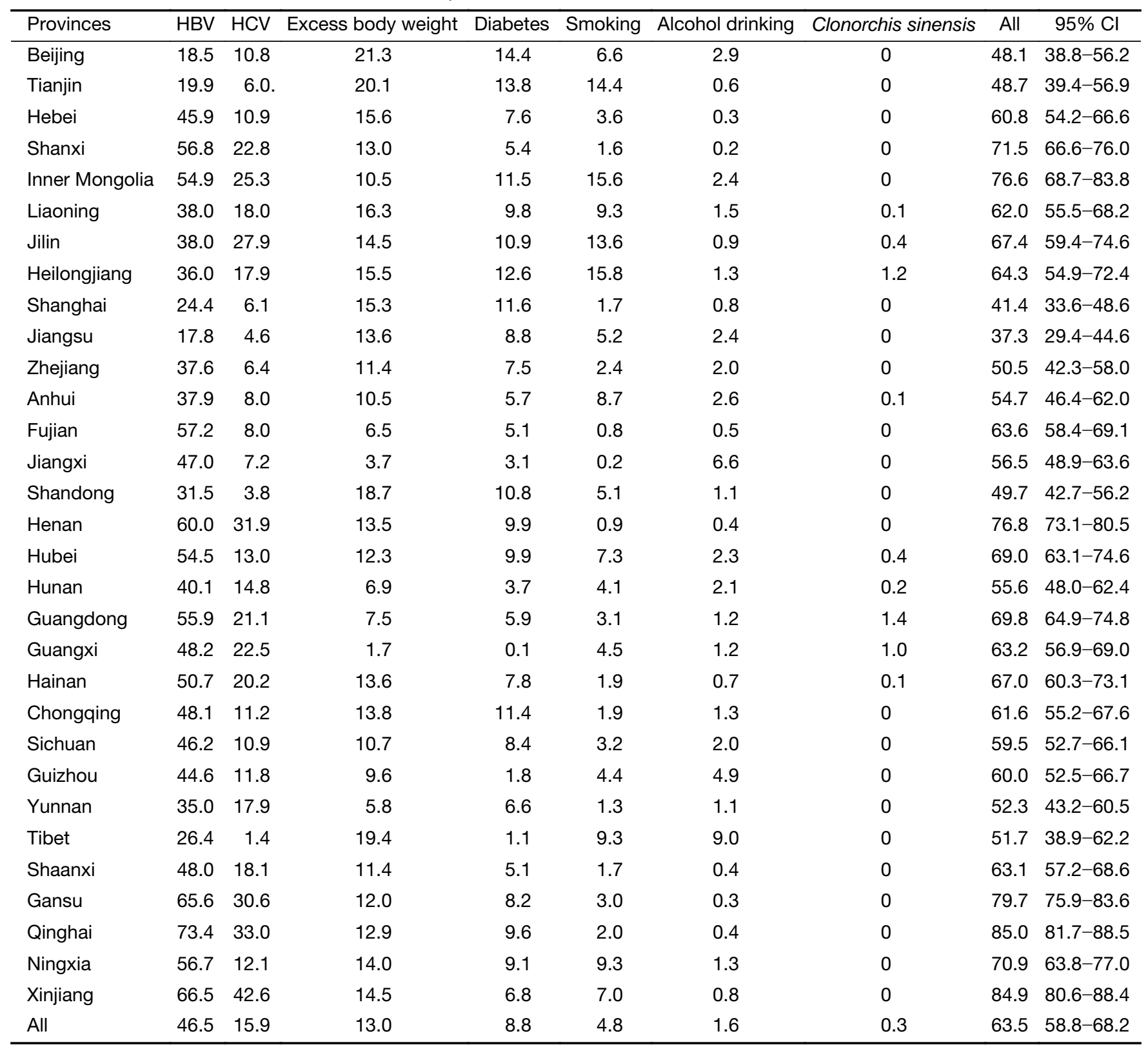

PAF, population attributable fraction; HBV, hepatitis B virus; $\mathrm{HCV}$, hepatitis $\mathrm{C}$ virus; $95 \% \mathrm{Cl}$, $95 \%$ confidence interval.

attributable to several well-established risk factors of liver cancer in China. We found that a substantial proportion of liver cancer deaths could be attributable to $\mathrm{HBV}$ and $\mathrm{HCV}$ infections. The study also reflected that there were significant variations in sex, age groups, and provincial distribution of the contribution of evaluated risk factors to liver cancer burden in China.

Chronic infection with HBV remains a major risk factor of liver cancer in China, although HBV infection rate showed a slight reduction. In China, the initiation of hepatitis $\mathrm{B}$ vaccination in newborns, paid by the central government, was launched in 2005 , contributing to a significantly decreased trend of the prevalence of $\mathrm{HBV}$ infection (18). However, more than 90 million people are chronically infected with HBV in 2017 in China, according to World Health Organization Global Hepatitis Report (19). The results highlight the urgent need to implement other effective measures to control the transmission of 


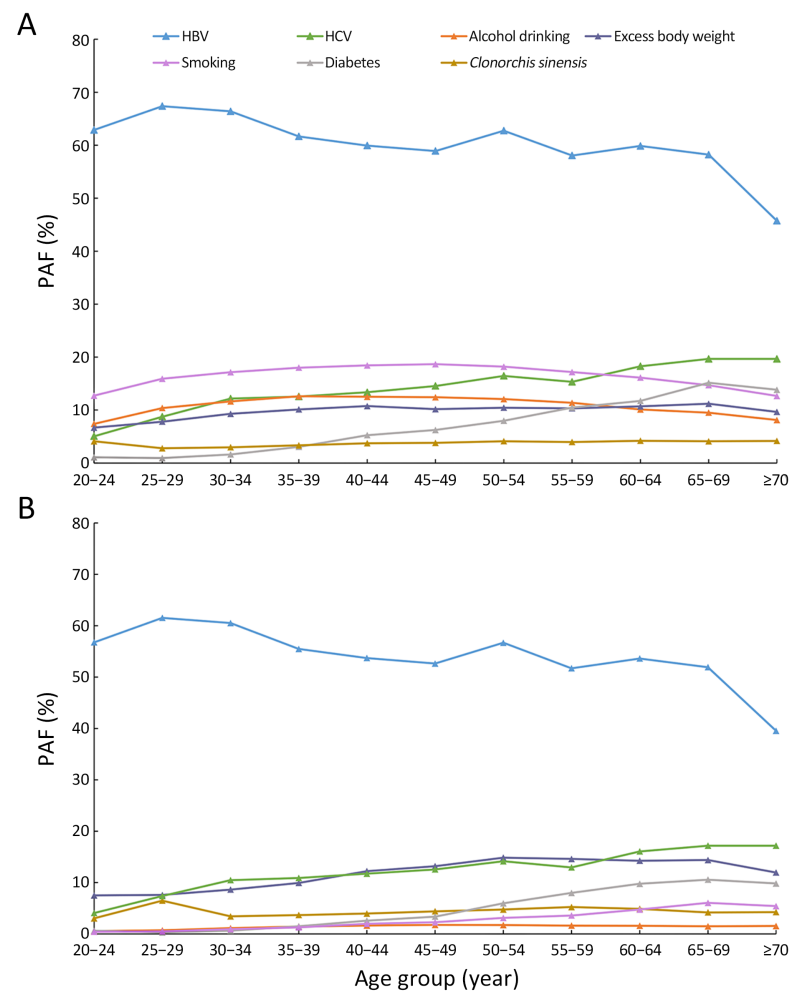

Figure 2 PAF for liver cancer by age group. (A) Male; (B) Female. $\mathrm{PAF}$, population attributable fraction; $\mathrm{HBV}$, hepatitis $\mathrm{B}$ virus; $\mathrm{HCV}$, hepatitis $\mathrm{C}$ virus.

$\mathrm{HBV}$ infection, excepting the massive coverage of vaccination. The pervasive lack of knowledge about HBV and its transmission route needs attention. In contrast, $\mathrm{HCV}$ infection is a relatively less critical factor of liver cancer in China. In 2006, the prevalence of HCV was estimated to be $0.43 \%$ nationally (12), and the proportion of liver cancer deaths attributable to $\mathrm{HCV}$ infection decreased dramatically in both genders, indicating the effective control of blood transfusions. We also noted that the PAF of HCV infection for liver cancer was increased with increasing age, supporting the conclusion that HCVrelated liver cancer cases are usually older (20). This may be due to the weak awareness of health care, dysfunction of the liver, and higher HCV infection among the elderly population. Similarly, the liver cancer burden attributable to infectious agents was higher in other countries. Studies conducted in the European Union (21) and Spain (22) found that $55 \%$ of all liver cancer deaths were attributable to $\mathrm{HBV}$ and $\mathrm{HCV}$, and $70 \%$ of liver cancer deaths were associated with $\mathrm{HCV}$ infections, respectively. Greater efforts should be paid to achieve the goal proposed by the Global Health Sector Strategy on viral hepatitis (21).
Excess bodyweight and diabetes are associated with a considerable burden of liver cancer, and their prevalence rates have increased substantially in recent years $(23,24)$. Dianne et al. estimated the proportion of liver cancer deaths attributable to excess bodyweight in Canada was $20.0 \%$ for males and $18.5 \%$ for females (25). Whereas PAF of excess bodyweight for liver cancer burden in our study was substantially lower than Dianne's estimates in both genders. The differences might be associated with the great impact of $\mathrm{HBV}$ infection, which may impair the role of excess bodyweight to liver cancer. Diabetes is related to metabolic change. A study has shown that participants with diabetes had a higher risk of liver cancer mortality, compared with those without diabetes (26). In the current study, the PAF of diabetes for liver cancer is relatively high, especially in females, which even exceeded the proportion attributable to smoking and alcohol drinking, showing the urgent need to effectively control the prevalence of diabetes. Our findings also suggested that the fractions of liver cancer burden attributable to diabetes were higher in older people than in young people. The results may be associated with higher prevalence in elderly people and females, compared with their counterparts (24).

Consistent with the estimates conducted by Fan et al., $\mathrm{HBV}$ also had the greatest PAF in both genders. The second greatest contributor to liver cancer burden was $\mathrm{HCV}$. Aflatoxin exposure contributed to the majority of liver cancer burden in 2005, second only to HBV and HCV. Aflatoxins are another important etiological factor of liver cancer in China, which have been classified as a Group 1 human carcinogen by the International Agency for Research on Cancer (27). Aflatoxins are produced primarily by the fungi Aspergillus flavus and Aspergillus parasiticus, which infect food crops such as maize, peanuts, groundnuts, and tree nuts (28). A population-based study has shown that if switching the dietary staple from maize to rice, $65 \%$ of the population attributable benefit could be gained in China (29). Thus, it is of significance to reduce aflatoxin contamination of food. Many provinces have suffered from both high aflatoxin exposure and high HBV prevalence, such as Jiangsu province. The interaction role of aflatoxin exposure and HBV may be associated with the high mortality rate of liver cancer in these provinces (28). It is difficult for us to estimate the detailed PAF of aflatoxin for liver cancer burden because data from provincial level aflatoxin exposure are limited in China and thus, we could not compare the differences of each province. For convenience, we collected information about the 
prevalence of aflatoxin from the high-quality meta-analysis with enough representativeness (28), and regarded it as a category variable referring to exposure to aflatoxin and not exposure to aflatoxin. We found that aflatoxin may play a role in about $12.6 \%$ of total liver cancer deaths, presenting a significant decrease compared with data in the study conducted by Fan et al. (Not shown in the result). Such favorable results might correlate with the reduction of contamination of dietary staples, the improvement of hygiene, and continuing economic growth (30).

PAFs for liver cancer showed significant sex, region, and age-specific variations. The major causes for liver cancer deaths in males were HBV, HCV, smoking, and diabetes. While in females, the corresponding order was HBV, $\mathrm{HCV}$, excess bodyweight, and diabetes. Smoking-related liver cancer deaths were higher in males than in females. Similar results were also obtained in Korea. And $23.5 \%$ of liver cancer deaths were attributable to tobacco use in males. However, only $6.1 \%$ of liver cancer deaths in females were attributable to smoking (31). This finding seems to be related to the low prevalence of smoking in females. For age-specific difference, in this study, the proportion of liver cancer deaths attributable to alcohol drinking, smoking, diabetes, and HCV infection was all increased with increasing age, particularly among those aged 60-69 years in both genders. This is probably because elderly people are the vulnerable population for cancer and are exposed to the studied risk factors easily. We also noted that the changing trends of smoking-attributable liver cancer deaths in males and females were different across age groups, presenting a decreasing trend with increasing age in males and an increasing trend with increasing age in females. In addition, due to the differences in geography, economic status, and dietary habits, the distribution of risk factors for liver cancer varies substantially in different provinces (32). Targeted policies should be made aiming at these differences.

There are some limitations to our study. First, aflatoxin exposure plays an important role in liver cancer burden. We failed to estimate the fraction of liver cancer burden attributable to aflatoxin exposure at the provincial level, due to the complex measurement of aflatoxin content. Second, the same RR estimations for different age groups and provinces were used, which might lower the accuracy to some extent. Third, because of the limitation of data, we did not take into account the combination role of selected risk factors, and we assumed that risk factors were independent of each other. Despite these limitations, our study has several strengths. First, data sources were credible. RR estimates were abstracted from large-scale and high-quality meta-analyses, and the prevalence of selected risk factors was abstracted from the representative survey. Thus, the potential bias of the calculation of PAF was minimized. Second, we presented the fraction for liver cancer burden attributable to several common risk factors of liver cancer by age group, sex, and provincial level. The present study using representative and extensive data could provide useful and targeted information for policy-makers.

\section{Conclusions}

Infectious factors continue to be the major causes of liver cancer burden in China, especially $\mathrm{HBV}$ infection. Preventive measures should be conducted according to gender, age groups, and regional differences. Further research should be concentrated on the combination role or interaction role of these evaluated risk factors.

\section{Acknowledgements}

This study was supported by the National Natural Science Foundation of China (No. 81974492); Non-profit Central Research Institute Fund of Chinese Academy of Medical Sciences (No. 2019PT320027); and Sanming Project of Medicine in Shenzhen (No. SZSM201911015).

\section{Footnote}

Conflicts of Interest: The authors have no conflicts of interest to declare.

\section{References}

1. Ferlay J, Ervik M, Lam F, et al. Global Cancer Observatory: Cancer Today. Lyon, France: International Agency for Research on Cancer. Available online: https://gco.iarc.fr/today

2. Baecker A, Liu X, La Vecchia C, et al. Worldwide incidence of hepatocellular carcinoma cases attributable to major risk factors. Eur J Cancer Prev 2018;27:205-12.

3. Yang JD, Hainaut P, Gores GJ, et al. A global view of hepatocellular carcinoma: trends, risk, prevention and management. Nat Rev Gastroenterol Hepatol 2019; 16:589-604.

4. Massarweh NN, El-Serag HB. Epidemiology of 
hepatocellular carcinoma and intrahepatic cholangiocarcinoma. Cancer Control 2017;24:1073274817729245.

5. Global Burden of Disease Liver Cancer Collaboration, Akinyemiju T, Abera S, et al. The burden of primary liver cancer and underlying etiologies from 1990 to 2015 at the global, regional, and national level: Results from the Global Burden of Disease Study 2015. JAMA Oncol 2017;3:1683-91.

6. Whiteman DC, Wilson LF. The fractions of cancer attributable to modifiable factors: A global review. Cancer Epidemiol 2016;44:203-21.

7. Fan JH, Wang JB, Jiang Y, et al. Attributable causes of liver cancer mortality and incidence in China. Asian Pac J Cancer Prev 2013;14:7251-6.

8. Chen W, Xia C, Zheng R, et al. Disparities by province, age, and sex in site-specific cancer burden attributable to 23 potentially modifiable risk factors in China: a comparative risk assessment. Lancet Glob Health 2019;7:e257-e69.

9. Charafeddine MA, Olson SH, Mukherji D, et al. Proportion of cancer in a Middle eastern country attributable to established risk factors. BMC Cancer 2017;17:337.

10. World Cancer Research Fund/American Institute for Cancer Research. Diet, nutrition, physical activity and cancer: a global perspective. Continuous Update project expert report 2018. Available online: http:// www.dietandcancerreport.org

11. Liang X, Bi S, Yang W, et al. Epidemiological serosurvey of hepatitis B in China--declining HBV prevalence due to hepatitis $B$ vaccination. Vaccine 2009;27:6550-7.

12. Chen YS, Li L, Cui FQ, et al. A sero-epidemiological study on hepatitis $\mathrm{C}$ in China. Zhonghua Liu Xing Bing Xue Za Zhi (in Chinese) 2011;32:888-91.

13. Li Y, Zhai F, Yang X, et al. Determinants of childhood overweight and obesity in China. Br J Nutr 2007;97:210-5.

14. Wu Y, Huxley R, Li L, et al. Prevalence, awareness, treatment, and control of hypertension in China: data from the China National Nutrition and Health Survey 2002. Circulation 2008;118:2679-86.

15. Li LM, Rao KQ, Kong LZ, et al. A description on the Chinese national nutrition and health survey in 2002. Zhonghua Liu Xing Bing Xue Za Zhi (in Chinese) 2005;26:478-84.
16. Lai YS, Zhou XN, Pan ZH, et al. Risk mapping of clonorchiasis in the People's Republic of China: A systematic review and Bayesian geostatistical analysis. PLoS Negl Trop Dis 2017;11:e0005239.

17. Lim SS, Vos T, Flaxman AD, et al. A comparative risk assessment of burden of disease and injury attributable to 67 risk factors and risk factor clusters in 21 regions, 1990-2010: a systematic analysis for the Global Burden of Disease Study 2010. Lancet 2012;380: 2224-60.

18. Shi JF, Cao M, Wang Y, et al. Is it possible to halve the incidence of liver cancer in China by 2050? Int J Cancer 2021;148:1051-65.

19. World Health Organization. Global hepatitis report, 2017. Available online: https://www.who.int/publications/ i/item/global-hepatitis-report-2017

20. Islami F, Dikshit R, Mallath MK, et al. Primary liver cancer deaths and related years of life lost attributable to hepatitis $\mathrm{B}$ and $\mathrm{C}$ viruses in India. Cancer Epidemiol 2016;40:79-86.

21. Mårdh O, Quinten C, Amato-Gauci AJ, et al. Mortality from liver diseases attributable to hepatitis $\mathrm{B}$ and $\mathrm{C}$ in the EU/EEA - descriptive analysis and estimation of 2015 baseline. Infect Dis (Lond) 2020; 52:625-37.

22. García-Fulgueiras A, García-Pina R, Morant C, et al. Hepatitis C and hepatitis B-related mortality in Spain. Eur J Gastroenterol Hepatol 2009;21:895-901.

23. Pang Y, Kartsonaki C, Turnbull I, et al. Diabetes, plasma glucose, and incidence of fatty liver, cirrhosis, and liver cancer: A prospective study of 0.5 million people. Hepatology 2018;68:1308-18.

24. Yang SH, Dou KF, Song WJ. Prevalence of diabetes among men and women in China. N Engl J Med 2010;362:2425-6.

25. Zakaria D, Shaw A. Cancers attributable to excess body weight in Canada in 2010. Health Promot Chronic Dis Prevn Can 2017;37:205-14.

26. Lam EKK, Batty GD, Huxley RR, et al. Associations of diabetes mellitus with site-specific cancer mortality in the Asia-Pacific region. Ann Oncol 2011;22:730-8.

27. Nazhand A, Durazzo A, Lucarini M, et al. Characteristics, occurrence, detection and detoxification of aflatoxins in foods and feeds. Foods 2020;9:644.

28. Liu Y, Chang CC, Marsh GM, et al. Population 
attributable risk of aflatoxin-related liver cancer: systematic review and meta-analysis. Eur J Cancer 2012;48:2125-36.

29. Chen JG, Egner PA, Ng D, et al. Reduced aflatoxin exposure presages decline in liver cancer mortality in an endemic region of China. Cancer Prev Res (Phila) 2013;6:1038-45.

30. Chen J, Zhu J, Wang G, et al. Qidong: a crucible for studies on liver cancer etiology and prevention.

Cite this article as: Cao M, Ding C, Xia C, Li H, Sun D, He S, Chen W. Attributable deaths of liver cancer in China. Chin J Cancer Res 2021;33(4):480-489. doi: 10.21147/j.issn.10009604.2021.04.05
Cancer Biol Med 2019;16:24-37.

31. Park S, Jee SH, Shin HR, et al. Attributable fraction of tobacco smoking on cancer using population-based nationwide cancer incidence and mortality data in Korea. BMC Cancer 2014;14:406.

32. Cui F, Zhang L, Yu C, et al. Estimation of the disease burden attributable to 11 risk factors in Hubei province, China: A comparative risk assessment. Int Environ Res Public Health 2016;13:944. 